\title{
Epigenome-wide association data implicate fetal/maternal adaptations contributing to clinical outcomes in preeclampsia
}

\author{
Teng Wang ${ }^{\ddagger}, 1$, Yuqian Xiang ${ }^{\ddagger 2,3}$, Xinyao Zhou' ${ }^{1}$ Xiaoguo Zheng ${ }^{2,3}$, Huijuan Zhang ${ }^{2}$, \\ Xiaojing Zhang ${ }^{4}$, Junyu Zhang ${ }^{2,3}$, Lin $\mathrm{He}^{5}$ \& Xinzhi Zhao*,2,3 \\ ${ }^{1}$ Children's Hospital \& Institutes of Biomedical Sciences, Fudan University, Shanghai, 200032, PR China \\ ${ }^{2}$ International Peace Maternity \& Child Health Hospital of China Affiliated to Shanghai Jiao Tong University, Shanghai, 200030, PR \\ China \\ ${ }^{3}$ Shanghai Key Laboratory of Embryo Original Diseases, Shanghai, 200030, PR China \\ ${ }^{4}$ Department of Obstetrics, Provincial Hospital Affiliated to Shandong University, Jinan, 250021, PR China \\ ${ }^{5}$ Bio-X Institutes, Key Laboratory for the Genetics of Developmental \& Neuropsychiatric Disorders (Ministry of Education), Shanghai \\ Jiao Tong University, Shanghai, 200030, PR China \\ *Author for correspondence: Tel.: +86 216407 0434; xzzhaoipmch@163.com \\ ${ }^{\ddagger}$ Authors contributed equally
}

\begin{abstract}
Aim: To investigate the changes of placental DNA methylome in preeclampsia (PE). Materials \& methods: We performed an epigenome-wide association study in a Chinese cohort and six published datasets consisting of 335 samples in total. Results \& conclusion: Numerous consistently hypomethylated probes were associated with early-onset PE in different populations, with 2125 reaching epigenome-wide significance. The validated probes were enriched for cytosine-phosphate-guanine dinucleotide (CpG) sites partially methylated and located in genes related to trophoblast fusion. The methylation levels of the validated probes were associated with clinical severity, while the intermediate samples showed antagonistic fetal/maternal outcomes. The DNA methylation patterns of PE and clinically relevant obstetrical syndromes suggested partially common pathophysiologies and complex maternal/fetal adaptive responses contributing to variable clinical outcomes.
\end{abstract}

First draft submitted: 6 March 2019; Accepted for publication: 3 May 2019; Published online:

16 May 2019

Keywords: DNA methylome $\bullet$ EOPE $\bullet$ fetal growth restriction

Preeclampsia (PE) is a pregnancy-induced hypertension syndrome that affects 3-5\% of all pregnancies, and is a leading cause of maternal and perinatal morbidity and mortality [1]. Although the pathophysiology of PE remains unclear, the syndrome is thought to occur in two stages with abnormal placentation leading to a maternal inflammatory response. The placenta plays a key role in the development of PE, and delivery of the placenta is the only effective treatment [2].

An increasing number of studies have revealed the etiological heterogeneity of PE. According to gestational age at diagnosis or delivery, PE can be classified as early ( $<34$ weeks) or late ( $\geq 34$ weeks) onset. Compared with late-onset $\mathrm{PE}$ (LOPE), early-onset PE (EOPE) is associated with more severe placental dysfunction, fetal growth restriction, and adverse maternal and fetal outcomes. However, whether EOPE and LOPE have different pathogenetic mechanisms or are gradations of the same underlying condition remains unclear [3]. Recently, transcriptome profiling showed that the multiple subtypes of $\mathrm{PE}$ [4], maternal predispositions, placental dysfunction and impaired immune tolerance were considered as predominant etiologies in different cases according to the gene expression patterns.

DNA methylation plays a key role in embryonic development and the regulation of gene expression [5]. Perturbations in DNA methylation patterns have been observed in various diseases and complex phenotypes [6]. A number of studies have been conducted to search for abnormalities in DNA methylation in PE. Some earlier studies measured the global genome methylation level in $\mathrm{PE}[7,8]$, while others quantitatively analyzed the methylation level in preselected candidate genes [9-11]. More recently, high-throughput platforms were used to identify differentially

Future Medicine 
Table 1. Sample clinical traits information

\begin{tabular}{|c|c|c|c|}
\hline Clinical traits & $\operatorname{PE}(n=30)^{\dagger}$ & Controls $(n=32)$ & p-value $\ddagger$ \\
\hline Maternal age (year) & $31.23 \pm 5.26$ & $30.09 \pm 3.97$ & 0.351 \\
\hline Gestational age (week) & $33.66 \pm 3.49$ & $39.10 \pm 2.26$ & $1.73 \times 10^{-8}$ \\
\hline Diastolic pressure $(\mathrm{mmHg})$ & $111.67 \pm 15.36$ & $82.58 \pm 12.62$ & $1.89 \times 10^{-6}$ \\
\hline Systolic pressure $(\mathrm{mmHg})$ & $163.47 \pm 22.01$ & $127.17 \pm 14.88$ & $6.00 \times 10^{-7}$ \\
\hline Baby weight (g) & $2037.14 \pm 931.71$ & $3391.25 \pm 634.62$ & $1.19 \times 10^{-7}$ \\
\hline Placenta weight (g) & $372.17 \pm 133.75$ & $510.77 \pm 88.74$ & 0.002 \\
\hline \multicolumn{4}{|c|}{$\begin{array}{l}\text { All data were shown in mean } \pm S D \text {. } \\
\dagger \text { In this study, PE was defined as: after } 20 \text { weeks of pregnancy, a new onset of: systolic pressure } \geq 140 \mathrm{mmHg} \text { or diastolic pressure } \geq 90 \mathrm{mmHg} \text {, urinal protein } \geq 0.3 \mathrm{~g} / 24 \mathrm{~h} \text {. } \\
\ddagger \mathrm{p} \text {-values were given by Mann-Whitney U-test; } \mathrm{p} \text {-values shown in bold reached significant level }(\mathrm{p}<0.05) \text {. } \\
\mathrm{PE} \text { : Preeclampsia. }\end{array}$} \\
\hline
\end{tabular}

methylated probes/regions (DMPs/DMRs) between PE and healthy specimens at the epigenome-wide scale [12-17]. In each study, hundreds of DMPs/DMRs were identified, supporting the abnormality of the DNA methylome in PE. However, these studies showed inconsistent results, reflecting the small sample sizes and lack of standardization of analytical approaches [18]. The lack of independent replication of key findings limits the understanding of epigenetic programming in $\mathrm{PE}$ and its biological role in placental function.

Here, we evaluated the effects of changes in the DNA methylome in PE placentas by analyzing data from six independent cohorts. We identified 2125 DMPs consistently associated with EOPE. These DMPs are hypomethylated in EOPE, which may be caused by downregulated de novo DNA methyltransferases. The methylation status of the DMPs clustered the placentas into several clinically relevant subgroups, which may have implications for the shared molecular pathology across multiple pregnancy complications and complex adaptive responses between mothers and fetuses.

\section{Materials \& methods}

Sample collection

All placental samples used in this study were collected from the Department of Obstetrics, Provincial Hospital Affiliated to Shandong University. Maternal and newborn characteristics were obtained from hospital medical records. The EOPE was diagnosed by new-onset gestational hypertension of at least 140/90 $\mathrm{mmHg}$ on two occasions at least $4 \mathrm{~h}$ apart accompanied by proteinuria $\geq 300 \mathrm{mg} /$ day from $24 \mathrm{~h}$ urine collection at $\geq 20$ weeks and $<34$ weeks of gestation. Normal controls were defined as pregnancies without PE, gestational hypertension, fetal growth restriction or preterm birth ( $<37$ weeks). The FGR was defined as fetal birth weight $<10$ th percentile for both fetal sex and gestational age at delivery. In total, 32 EOPE and 30 normal controls were recruited in this study (Table 1). All participants are Han Chinese in origin.

All placentas were collected immediately after caesarean section. The placental samples at the chorionic plate were separately removed from each quadrant along with the central portion in the placental disc. Approximately $1 \mathrm{~cm}^{3}$ fragments were dissected from the placenta after removing maternal blood by vigorous washing with phosphatebuffered saline. The tissues were maintained in centrifuge tubes with RNA later (Ambion, Inc., TX, USA) and frozen at $-80^{\circ} \mathrm{C}$. The study protocols were approved by the Ethics Review Committee of Fudan University and conducted according to the principles of the Declaration of Helsinki. All participants provided written informed consent before participation.

\section{DNA methylome profiling \& differential methylation analysis}

A total of 22 EOPE and 20 normal placental samples were used for DNA methylome profiling and the result was named CN dataset. Genomic DNA was extracted with a QIAGEN DNAeasy blood \& tissue kit (Hilden, Germany) from the placental tissue. Bisulfate conversion was performed using an EZ-96 DNA Methylation-Gold ${ }^{T M}$ Kit (Zymo Research Corp., CA, USA). The converted DNA templates were then used for PCR amplification and the products were dissolved and dispensed on the Illumina Infinium HumanMethylation450 (450k) BeadChip and assayed on the Illumina HiScanSQ system (CA, USA). The results are available in the GEO repository with accession number GSE125605. 
To analyze the raw data obtained using the 450k chip, minfi package in $\mathrm{R} 3.1 .3$ was used and the subsetquantile within array normalization method was used to normalize the results. At each cytosine-phosphate-guanine dinucleotide (CpG) site, the $\beta$ value and $\mathrm{M}$ value were given by minfi [19] to describe the methylation level. The $\beta$ value indicates the methylation percentage of each $C p G$ site, while the $M$ value was defined as $\log _{2} \frac{\beta}{1-\beta}$. We performed the following steps for data filtering: the probes located on chromosomes $\mathrm{X}$ and $\mathrm{Y}$ were excluded; the probes with single-nucleotide polymorphisms at the CpG site or within ten base pairs of the CpG site were excluded; and the probes with null (missing $\beta$ value) $\mathrm{CpG}$ sites were excluded; the probes on which the ratio of samples with detection p-values $>0.01$ was larger than $5 \%$ were excluded. The DMPs and DMRs were evaluated by ChAMP package [20] under R 3.3.1. We set the threshold for EWS as $\mathrm{p}<1 \times 10^{-7}$ to provide a conservative Bonferroni correction for the number of markers tested [21].

Technical validation of differentially methylated probes with MassARRAY EpiTYPER

Primers for MassARRAY EpiTYPER were designed with EpiDesigner (www.epidesigner.com). Samples treated with the Methylation-Gold ${ }^{\text {TM }}$ Kit were amplified by PCR, cleaved with restriction endonuclease, conditioned with resin and dispensed into SpectroCHIPs (Sequenom, CA, USA). At least two replicates were evaluated for each reaction. The SpectroCHIPs were evaluated on a SEQUENOM MassARRAY Workstation (Sequenom, CA, USA), and the results were analyzed with Quantitative Methylation Analysis software.

System review of epigenome-wide DNA methylation studies on PE \& meta-analysis

We performed a search of literature published through August 2017 in PubMed to identify all articles reporting differential DNA methylation in PE. In the 147 identified studies by using the keywords 'DNA methylation' and 'PE', Among the genome-wide studies, 450k was used in six studies; $450 \mathrm{k}$ was also used for our Chinese cohort. However, the sampling procedures differed in the two studies [17,22] and thus, these studies were excluded from further analysis.

We downloaded the DNA methylome datasets for the four remained studies from the Gene Expression Omnibus database (Supplementary Table 1). Because more significant changes in DNA methylation were observed in EOPE, 53 EOPE cases and 65 controls from the CN dataset, CA dataset (GSE44667) [12] and NL dataset (GSE103253) [15] were used for meta-analysis. Meta-analysis was performed using the metafor package [23] under R 3.1.3. The standardized mean difference of the $M$ value was used as the combined effect size to test the association for each probe. The mean difference in the $\beta$ value was used to describe the difference in the methylation level $\left(\Delta \beta_{\text {meta }}\right)$. Heterogeneity across studies was evaluated using the Q-test. An inverse variance method was used to calculate the fixed-effect estimate, and the DerSimonian \& Laird method was used to measure the random-effect magnitude.

The CA2 dataset (GSE98224) [24] and CAplus dataset (GSE100197) [16] were available online in 2018 [16,24] (Supplementary Table 1). However, nearly all EOPE and preterm control samples in the CAplus dataset have been used in the CA dataset. The CA2 dataset, US dataset (GSE57767) [13] and AU dataset (GSE75196) [14] were used to further validate the results of meta-analysis according to the p-value and $\Delta \beta$.

\section{Gene ontology enrichment analysis}

Gene Ontology (GO) analysis was conducted using the Database for Annotation, Visualization and Integrated Discovery (DAVID) [25] (http://david.ncifcrf.gov) and topGO [26] package under R 3.1.3.

\section{Clustering analysis}

Clustering was conducted using the heatmap function under R 3.1.3.

\section{Gene expression assay}

Total RNA was extracted from placental tissue using the Ambion mirVana ${ }^{T M}$ PARIS ${ }^{T M}$ Kit (CA, USA) according to the manufacturer's instruction. RNA $(1 \mu \mathrm{g})$ was reverse-transcripted into cDNA with the RevertAid First Strand cDNA Synthesis Kit (Thermo Fisher Scientific, MA, USA). The qPCR was performed using SYBR Green Master Mix (Roche, Basel, Switzerland) with $10 \mathrm{ng}$ cDNA in a $10 \mu \mathrm{l}$ volume using the ABI Prism 7900HT Fast Real-Time PCR System (Life-Technologies Applied Biosystems, CA, USA) and the results were normalized using the reference gene GAPDH. The result of each reaction was determined as the mean value of three replicates. Primers used in this study are shown in Supplementary Table 2. 
Table 2. Top 20 differentially methylated probes in 464 most significant differentially methylated probes in result of CN DATA.

\begin{tabular}{|c|c|c|c|c|c|c|}
\hline Probe ID & Gene & Feature & $\boldsymbol{\beta}_{c^{\dagger}}$ & $\boldsymbol{\beta}_{\mathbf{P}}^{\dagger}$ & $\Delta \beta$ & p-value $\ddagger$ \\
\hline cg26940261 & C12orf75 & Body & 0.19 & 0.12 & -0.07 & $3.03 \times 10^{-13}$ \\
\hline cg25247520 & PVT1_MIR1204 & TSS200 & 0.49 & 0.36 & -0.13 & $2.29 \times 10^{-12}$ \\
\hline cg12446045 & NA & IGR & 0.69 & 0.53 & -0.16 & $4.70 \times 10^{-12}$ \\
\hline $\operatorname{cg} 25691553$ & NDUFAF3 & TSS200 & 0.21 & 0.1 & -0.11 & $5.46 \times 10^{-12}$ \\
\hline cg00970435 & $A R S G$ & Body & 0.29 & 0.16 & -0.14 & $7.73 \times 10^{-12}$ \\
\hline ch.11.1352825R & NA & IGR & 0.09 & 0.04 & -0.05 & $7.99 \times 10^{-12}$ \\
\hline ch.3.946759R & FYCO1 & Body & 0.12 & 0.07 & -0.05 & $1.17 \times 10^{-11}$ \\
\hline ch.20.1019750F & SULF2 & $5^{\prime}$ UTR & 0.24 & 0.16 & -0.08 & $1.66 \times 10^{-11}$ \\
\hline cg07451391 & NA & IGR & 0.85 & 0.74 & -0.11 & $1.75 \times 10^{-11}$ \\
\hline cg17640147 & NA & IGR & 0.87 & 0.78 & -0.09 & $2.69 \times 10^{-11}$ \\
\hline ch.10.14508944R & NA & IGR & 0.08 & 0.03 & -0.05 & $3.14 \times 10^{-11}$ \\
\hline cg09857826 & PVT1 & Body & 0.74 & 0.57 & -0.17 & $3.83 \times 10^{-11}$ \\
\hline cg14458575 & NA & IGR & 0.24 & 0.14 & -0.1 & $3.85 \times 10^{-11}$ \\
\hline $\operatorname{cg} 25565850$ & OBSL1 & Body & 0.23 & 0.16 & -0.07 & $4.54 \times 10^{-11}$ \\
\hline cg06682024 & MERTK & Body & 0.2 & 0.14 & -0.06 & $4.77 \times 10^{-11}$ \\
\hline cg20371401 & $\mathrm{C2}$ & 1stExon & 0.49 & 0.39 & -0.1 & $6.61 \times 10^{-11}$ \\
\hline cg00091483 & PEBP1 & Body & 0.18 & 0.09 & -0.09 & $8.97 \times 10^{-11}$ \\
\hline cg15564619 & SKI & Body & 0.2 & 0.11 & -0.09 & $9.26 \times 10^{-11}$ \\
\hline cg25073998 & NA & IGR & 0.47 & 0.34 & -0.13 & $1.08 \times 10^{-10}$ \\
\hline cg16583884 & $C C D C 68$ & TSS200 & 0.33 & 0.19 & -0.14 & $1.12 \times 10^{-10}$ \\
\hline
\end{tabular}

Cell culture, transfection

The siRNA for inhibiting the expression of CMIP, together with negative controls, were synthesized by Invitrogen (Invitrogen Stealth ${ }^{\top \mathrm{T}}$, CA, USA). CMIP inhibitor sequence: 5'-GCUGAACCUGUGGUCCACUCAGUUU-3'.

HTR8/SVneo cells were maintained in RPMI 1640 media (Gibco, NY, USA) containing 10\% fetal bovine serum (Sijiyuan Inc., Shanghai, China) and 1\% penicillin-streptomycin (Gibco) in 24 -well plates at $37^{\circ} \mathrm{C}$ and $5 \%$ $\mathrm{CO}_{2}$. The cells were approximately $50 \%$ confluent before transfection.

The siRNA/liposome complex was produced immediately before infection. The siRNA was diluted in OptiMEM (Gibco) to a final concentration of $1 \mu \mathrm{M}$, and each well of a 24-well plate was transfected with 5 pmol siRNA and $1.25 \mu$ l Lipofectamine ${ }^{T M} 3000$ (Invitrogen, CA, USA). Total RNA was extracted with TRIzol reagent (Ambion, CA, USA).

\section{Results}

Overview of differentially methylated probes associated with EOPE in a Chinese cohort

After implementing the filtering steps described in the methods, 471,481 probes were included in the analyses. A total of 464 probes reached epigenome-wide significance (EWS) $\left(\mathrm{p}<1 \times 10^{-7}\right.$ and $\left.|\Delta \beta|>0.05\right), 459(98.9 \%)$ of which were hypomethylated in EOPE in the Chinese cohort. The top 20 significant probes are shown in Table 2 and all probes are listed in Supplementary Table 3. Several probes in $P V T 1$ were highly significantly hypomethylated in PE. We carried out technical validation of nine probes with the MassARRAY platform using more samples in the Chinese cohort, and all probes were significantly hypomethylated in EOPE placentas. Their neighbor regions showed correlated changes in DNA methylation (Supplementary Figure 1A-F).

\section{Comparison of results between the $\mathrm{CN}$ dataset \& previous reports}

In general, the results in our $\mathrm{CN}$ dataset showed poor consistency with the results of previous studies described in Supplementary Table 1. Nearly all (98.9\%) probes showing significant associations were hypomethylated in EOPE in the $\mathrm{CN}$ dataset. In the CA dataset, among the 282 significant probes, $74.5 \%$ were hypomethylated in EOPE. In the NL dataset, 467 (67\%) were hypomethylated within 697 significant probes. However, only 24 of 229 (10.5\%) 
were hypomethylated in PE cases with preterm birth in the US dataset, and 99 of 362 (27.3\%) significant DMRs were hypomethylated in LOPE cases in the AU dataset (Supplementary Table 4). Even the studies showed similar directions of methylation changes, few differentially methylated probes were recurrently identified. For example, only two probes overlapped among the 464 probes in the $\mathrm{CN}$ dataset and 282 in the CA dataset.

The lack of replicated probes between studies may be caused in part by different thresholds of association. We observed that stricter criteria of association resulted in a higher proportion of hypomethylated probes, as shown in Supplementary Table 4. When our threshold of EWS $\left(\mathrm{p}<1 \times 10^{-7}\right.$ and $\left.|\Delta \beta|>0.05\right)$ was used, 765 of 890 (86.0\%) DMPs were hypomethylated in EOPE in the CA dataset, 368 of 376 (97.9\%) DMPs were hypomethylated in EOPE in the NL dataset and five of six (83.3\%) DMPs were hypomethylated in EOPE in the AU dataset. However, no DMPs reached the standard for significance in the US dataset.

\section{Meta-analysis combining DNA methylome data from three independent EOPE studies}

To improve our power to test the associations in probes with weak effect sizes, 118 samples (53 EOPE and 65 controls) from three datasets (CN, CA and NL [CN dataset is the 450k result of our study with Chinese Han samples. CA dataset is the 450k result of Blair et al. with Canadian samples (GSE44667), NL dataset is the 450k result of Herzog et al. with Netherlandish samples (GSE103253)]) were used for meta-analysis. Numerous hypomethylated CpGs were observed in EOPE placentas, with 2307 probes reaching EWS ( $p<1 \times 10^{-7}$ in metaanalysis and $|\Delta \beta|>0.05$ in each study). The strongest finding $\left(\mathrm{p}=1.17 \times 10^{-23}\right)$ was observed for $\operatorname{cg} 10246581$ within an intron of $C M I P$, which contained three of the top ten significant probes.

Two additional datasets were available online in 2018 [16,24] (Supplementary Table 1). The CA2 dataset consisted of independent PE and control samples, while nearly all EOPE and preterm control samples in the CAplus dataset were used as CA dataset. So we only validated these 2307 probes in the CA2 dataset, with 2177 (94.4\%) probes showing a nominal association ( $\mathrm{p}<0.05$ and $|\Delta \beta|>0.05$ in the same direction). The reproducibility rate was much higher than those reported previously [14,16], which was only approximately $1 / 3$. A total of 2125 of the 2177 probes were hypomethylated in PE placenta in the US and AU datasets, and were considered as validated DMPs. The top 20 significant probes are shown in Table 3 and all the probes used are listed in Supplementary Table 5.

The 2125 DMPs were distributed in all autosomes (Figure 1A). In general, they showed mild hypomethylation levels in EOPE placentas: only 247 (11.62\%) of the DMPs had $\Delta \beta_{\text {meta }}<-0.1,4(0.19 \%)$ showed a value less than -0.15 , and none were less than -0.2. The DMPs showed strong enrichment of intermediate levels of methylation (Figure 1B).

\section{Validated DMPs associated with EOPE are enriched in genes related to trophoblast fusion}

A total of 1457 of 2125 validated DMPs were mapped to 1031 genes. Most of these genes are expressed in termed placenta $(864 / 1031,83.8 \%$, based on GSE44711). The annotated genes were subjected to GO analysis and FDR correction was applied to p-values, and ten and three significant GO terms (corrected $\mathrm{p}<0.05$, foldenrichment $>1.5$ ) were identified according to cellular component and molecular function (Supplementary Table 6). Genes related to cell migration, cell-cell adhesion, cellular membranes and transmembrane complexes were highly enriched, suggesting dysfunction in trophoblast fusion [27].

\section{Hierarchical clustering placentas with validated DMPs}

Based on the 2125 validated DMPs associated with EOPE, we performed hierarchical clustering of all datasets and identified four subgroups (Figure 2A-G). Consistent with the results showing that all DMPs were hypomethylated in EOPE compared with in controls, most term controls belonged to a subgroup with the highest DNA methylation level; this subgroup was named as EP0, reflecting no epigenetic programming related to EOPE. However, some preterm controls, LOPE, isolated FGR and even few EOPE samples belonged to EP0. The EOPE samples clustering to EP0 showed high gestational ages and averaged sized infants.

We named the other three groups as EP1, EP2 and EP3 because they were hypomethylated compared within EP0, with EP3 showing the lowest methylation level. All samples clustered in EP3 were diagnosed with PE combined with FGR and/or being very early-onset. EOPE clustered to EP3 were associated with a lower gestational age (30.23 vs 32.79 weeks; $\mathrm{p}=0.003$ ) and higher risk of FGR (RR = 1.86; 95\% CI: 1.26-2.76; $\mathrm{p}=0.002)$ Compared with non-EP3 EOPE samples in the CN, CA and CA2 datasets. Interestingly, we could not cluster EP3 from the NL dataset, as few EOPE samples (7.7\%) combined with FGR. These results suggest that EP3 represented a subgroup of PE with the worst placental function. However, few LOPE samples coexisting with FGR were clustered 


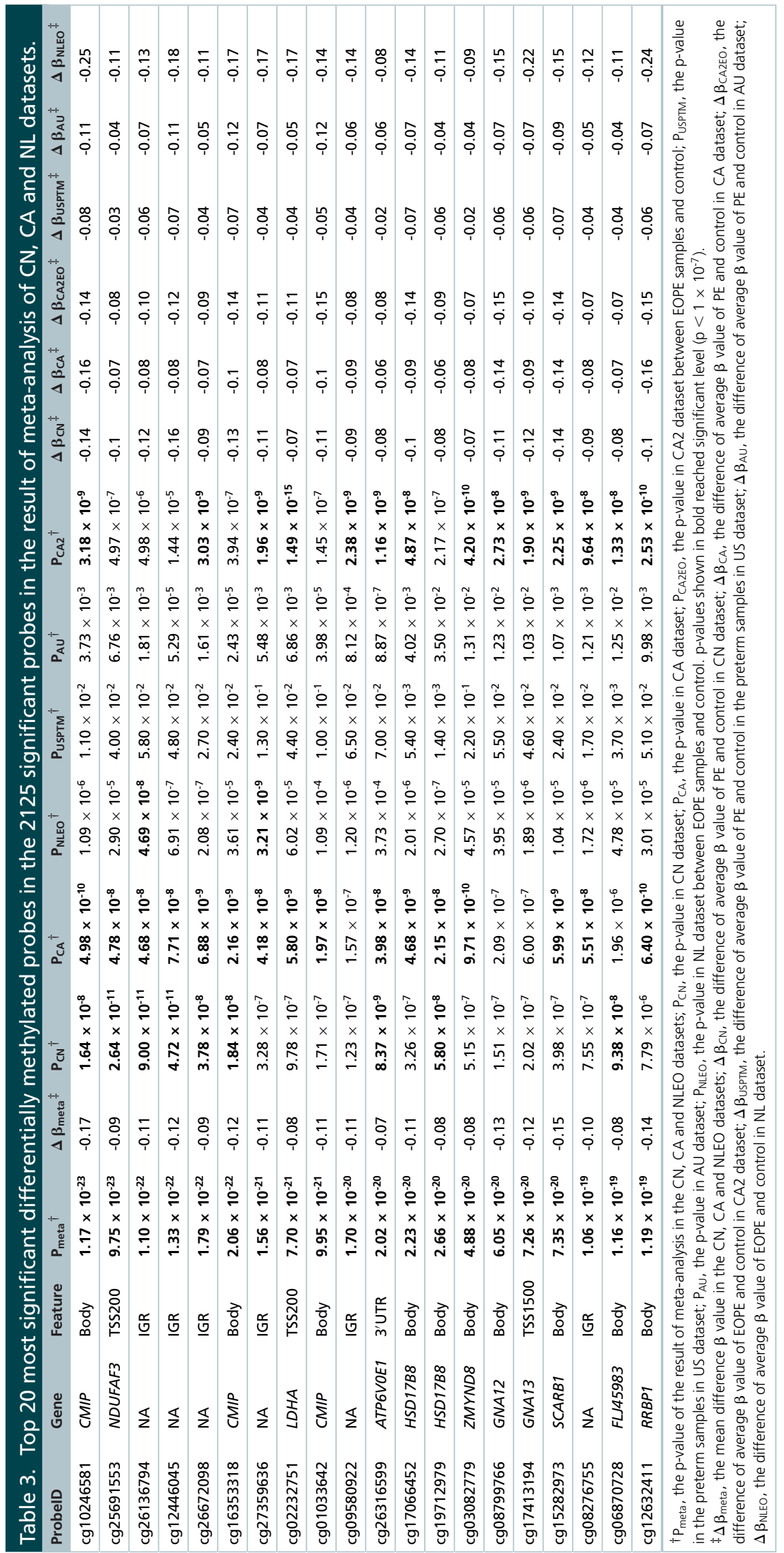




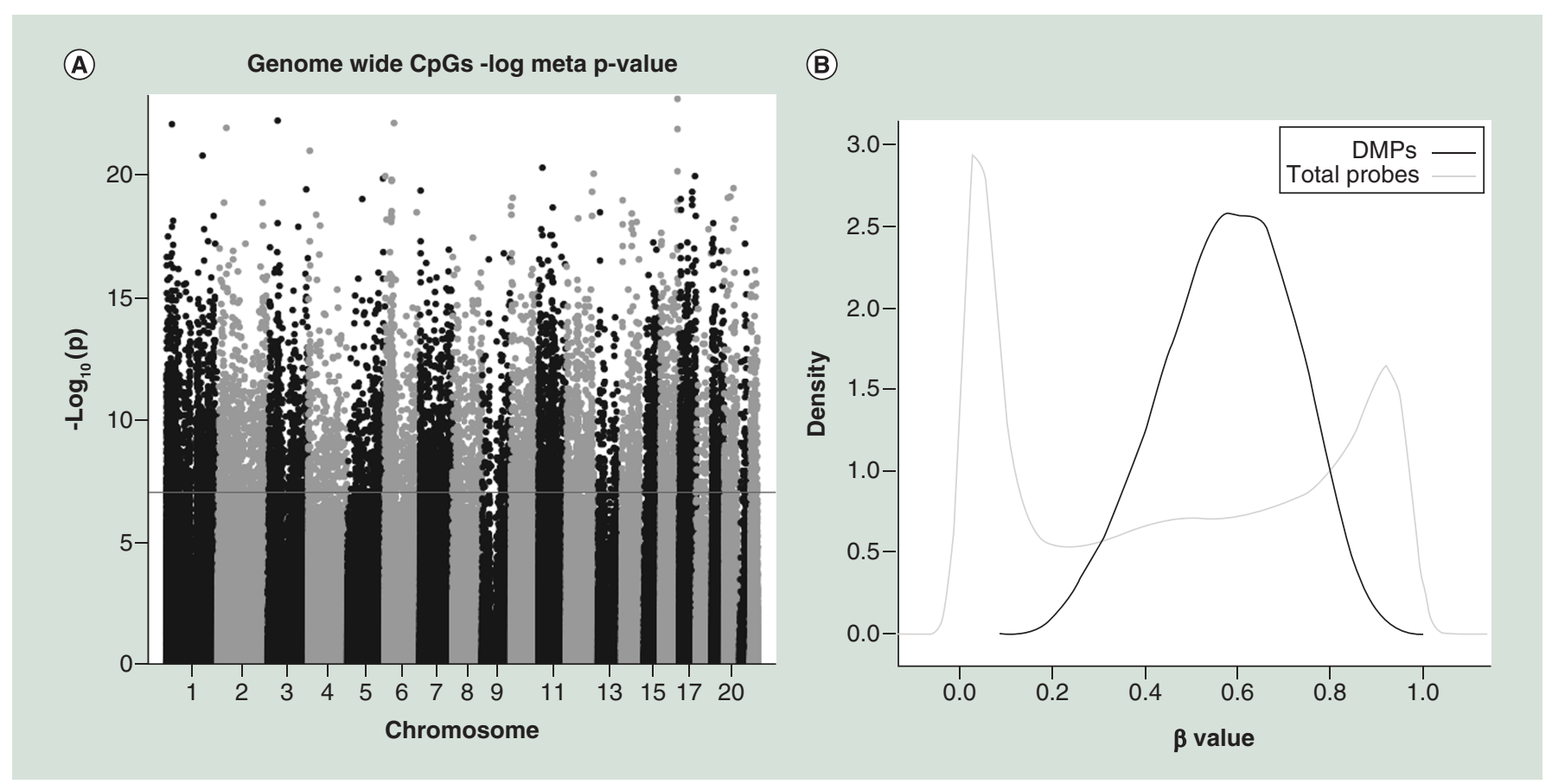

Figure 1. Distribution of the significant hypomethylated differentially methylated probes in the meta-analysis result. (A) Manhattan plot of the 2125 significant DMPs on the whole genome. (B) Density plot of the $\beta$ value of the 2125 significant DMPs, together with the $\beta$ value of the total $\mathrm{CpGs}$ detected by probes, on CN dataset.

CpG: Cytosine-phosphate-guanine dinucleotide; DMP: Differentially methylated probe.

into EP3. The EP2 consisted of most EOPE, a few LOPE, isolated FGR, or preterm controls, and very few term controls. Taken together, EP3 and EP2 (named EP2 ${ }^{+}$) included most EOPE samples, suggesting canonical EOPE-fashioned epigenetic programming. The remaining EP1 showed an intermediate epigenetic feature and most heterogeneous clinical features. Interestingly, samples in EP1 showed antagonistic maternal/fetal outcomes, including mild maternal syndromes which were associated with poorer fetal growth and vice versa.

In a previous study, 599 probes were used to cluster samples in the CAplus and CA2 datasets, with three DNA methylation clusters identified (MC1-MC3) [16]. Although only 220 of the 599 probes overlapped with our 2125 DMPs, MC1 which represented EOPE showed very a similar composition as EP2 ${ }^{+}$in the CAplus dataset, as 23 samples overlapped among the $24 \mathrm{MC} 1$ and $26 \mathrm{EP}^{+}$samples. A total of 20 samples overlapped among the 27 MC3 and 31 EP0 samples. The main discordance was observed in the MC2 or EP1 samples, which represented intermediate methylation groups. Importantly, after removing the overlapped DMPs, the remaining 1905 DMPs resulted in very similar hierarchical clusters (Supplementary Figure 2). These results suggested the overlapped DMPs might not critical.Clustering of the CA2 dataset showed less consistency, as 11 of the 12 EP1 samples were clustered in MC1. Epigenetic programming in EOPE may be continuous rather than sectionalized. Interestingly, four of the five EOPE samples clustered into EP1 and MC1 were diagnosed with chronic hypertension. We found that the $12 \mathrm{PE}$ samples with chronic hypertension showed weaker epigenetic programming compared with the remaining $22 \mathrm{PE}$ samples ( $\mathrm{p}=0.03$, Mann-Whitney $\mathrm{U}$ test) in the CA2 dataset.

The CA2 dataset was previously clustered based on transcriptome profiling [4]. We compared the results of transcriptome clusters with those of our epigenetic clusters. The transcriptome cluster 1 representing healthy placentas showed the lowest proportion of EP2 ${ }^{+}(10.5 \%)$, while the transcriptome cluster 2 representing canonical EOPE showed the highest proportion (78.9\%). This result supported the correlation between the transcriptional and epigenetic clusters. Additionally, the transcriptome cluster 3 associated with maternal antifetal rejection showed fewer epigenetic changes compared with cluster3, with $40 \%$ samples classified as $\mathrm{EP} 2^{+}$. This result suggests that epigenetic programming is relieved by restricting fetus growth. 


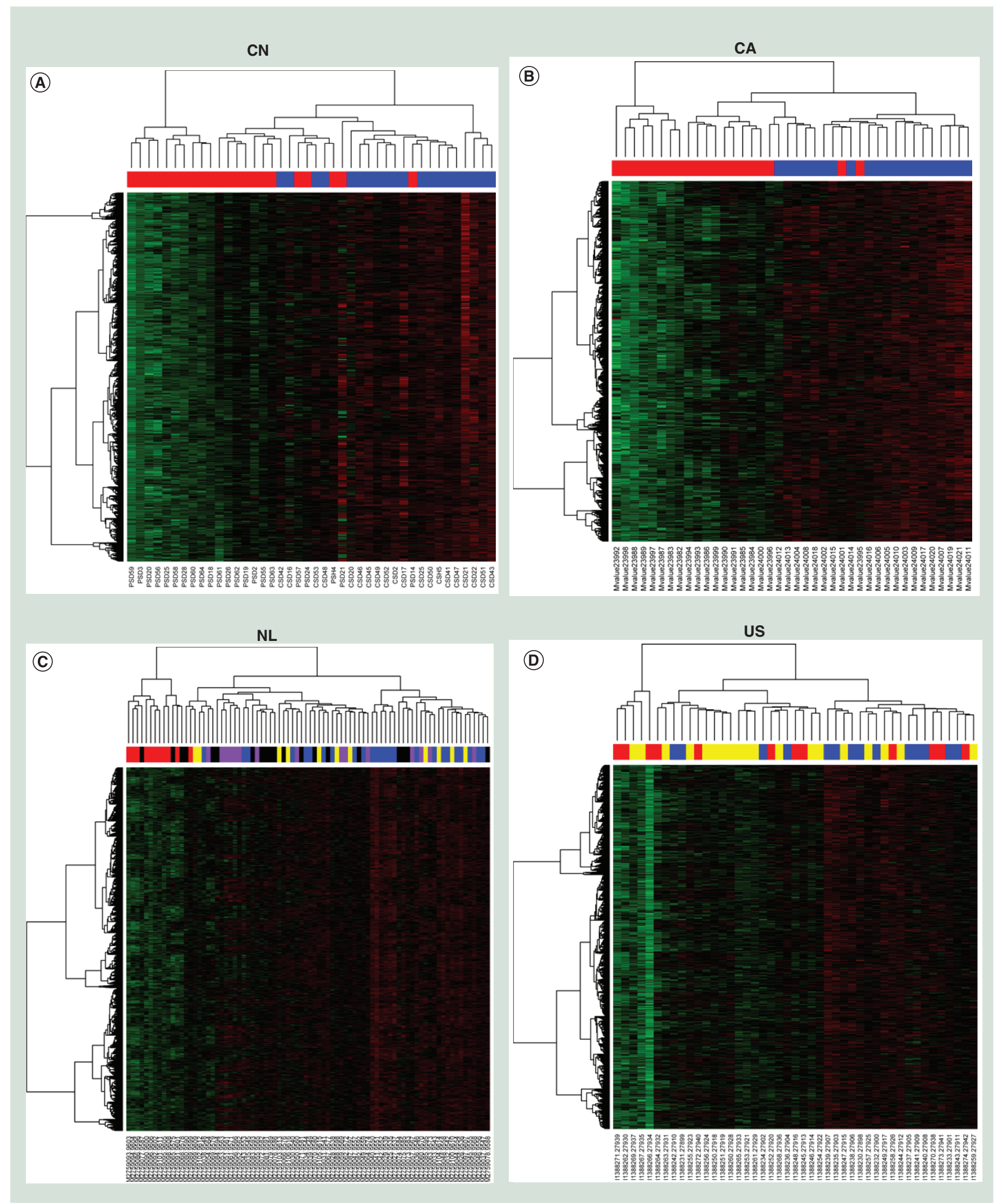

Figure 2. M values of $2125 \beta$ clustered in six datasets. (A) 42 samples of CN. (B) 40 samples of CA. (C) 82 samples of NL. (D) 45 samples of US. (E) 24 samples of AU. (F) 48 samples of CA2. (G) 102 samples of CAplus. Red = early-onset preeclampsia; black = fetal growth restriction; purple $=$ preterm birth; yellow $=$ late-onset preeclampsia; blue $=$ normal. 


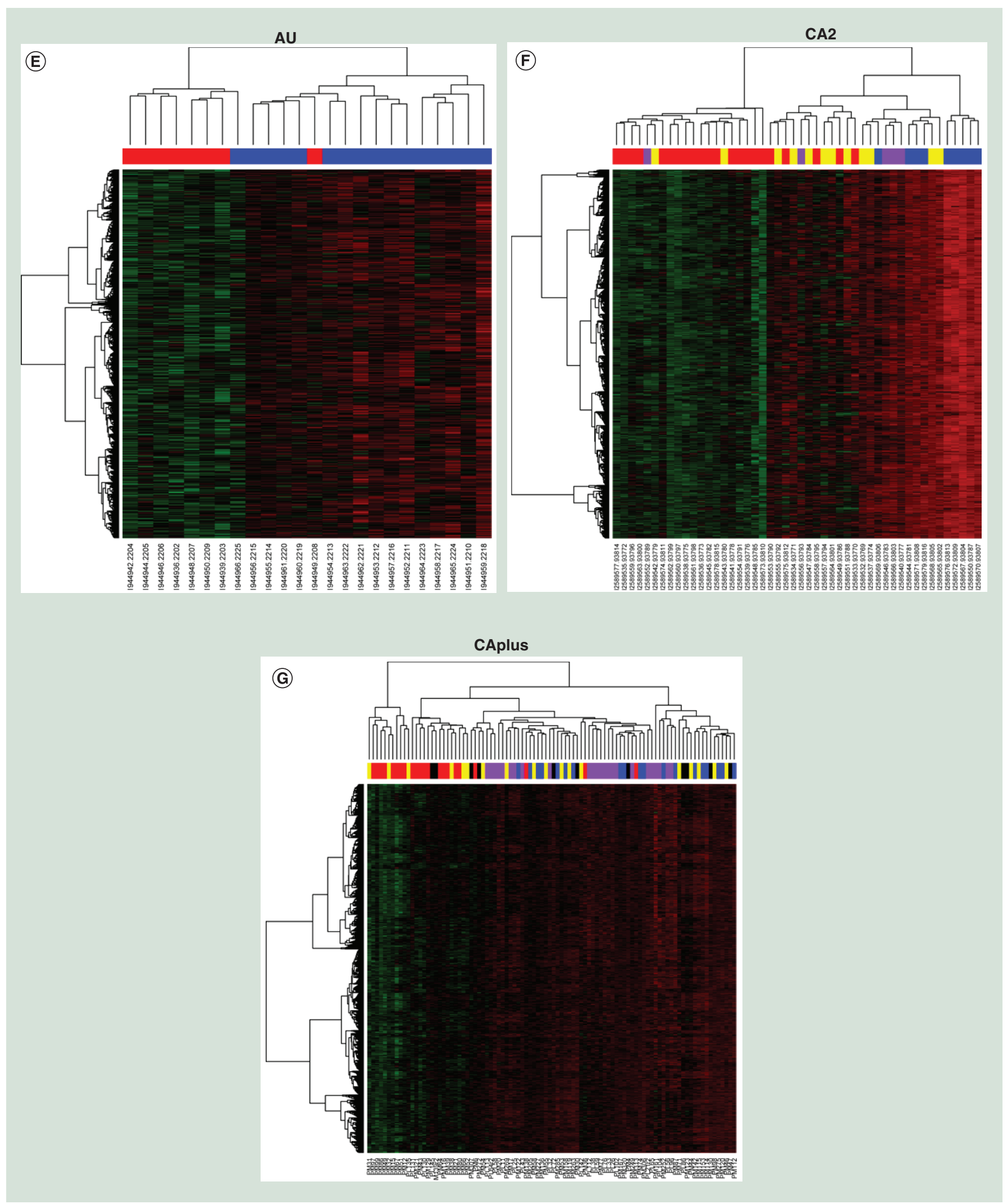

Figure 2. $M$ values of $2125 \beta$ clustered in six datasets (cont.). (A) 42 samples of CN. (B) 40 samples of CA. (C) 82 samples of NL. (D) 45 samples of US. (E) 24 samples of AU. (F) 48 samples of CA2. (G) 102 samples of CAplus. Red = early-onset preeclampsia; black = fetal growth restriction; purple = preterm birth; yellow = late-onset preeclampsia; blue = normal. 
Hypomethylation of DMPs is associated with downregulated de novo DNA methyltransferases The expression of three methyltransferases and three TET methylcytosine dioxygenases in placentas was measured by qPCR. In EOPE placenta, DNMT3A and DNMT3B showed significantly lower mRNA levels (DNMT3A: $\mathrm{p}=8.8 \times 10^{-4}$, fold change $=0.46 ; D N M T 3 B: \mathrm{p}=0.015$, fold change $=0.50$, corrected for eight tests using Bonferroni correction), while DNMT1 and TET1, TET2 and TET3 showed no significant changes (Figure 3A). The expression of $D N M T 3 A$ was significantly lower in EP3 and $D N M T 3 B$ was significantly lower in the EP1, EP2 and EP3 comparing with EP0 (DNMT3A expression: EP1: $\mathrm{p}=0.06$; EP3: $\mathrm{p}=7.1 \times 10^{-4} ;$ DNMT3B expression: $E P 1: p=3.4 \times 10^{-4} ; E P 2: p=6.0 \times 10^{-4} ; E P 3: p=0.015$; Figure 3B, Bonferroni corrected for eight tests).

\section{Validated DMPs \& related gene functions}

We evaluated the expression of ten genes containing the most significant DMPs. Although gene expression was poorly correlated with DNA methylation in the placenta in previous reports, we detected downregulated expression of CMIP, GNA12 and AGO2 in EOPE placenta (CMIP: $\mathrm{p}=0.025 ; G N A 12: \mathrm{p}=0.008 ; A G O 2: \mathrm{p}=0.011$, Bonferroni corrected for ten tests) (Figure 4A-D). We noticed that these downregulated genes were hypomethylated in gene body region. The DNA methylation in this region is often correlated with gene expression [28].

Three DMPs on the gene body of CMIP among the top ten significant probes in meta-analysis and gene expression were downregulated in EOPE. We inhibited CMIP expression by infecting the HTR8/SVneo cell line with CMIP inhibitor siRNA. We observed increased expression of VEGFA, VEGFC, HIFIA and RelA, which were associated with hypoxia and imbalance of angiogenic/antiangiogenic factors in PE $\left(V E G F A: \mathrm{p}=1.1 \times 10^{-5}\right.$; VEGFC: $\mathrm{p}=0.005 ;$ HIF1A: $\mathrm{p}=1.4 \times 10^{-4} ;$ RelA: $\mathrm{p}=2.2 \times 10^{-4} ;$ CMIP: $\mathrm{p}=2.7 \times 10^{-9}$, Bonferroni corrected for five tests) (Supplementary Figure 3A-E). This result supports a role for CMIP in the pathophysiology of PE.

\section{Discussion}

DNA methylation changes in EOPE placentas

In the present study, we conducted an epigenome-wide association study of EOPE in a Chinese Han cohort and combined our results with other data obtained from a public database. We detected numerous mild to moderate DNA methylation changes in EOPE placentas compared within healthy controls. All DMPs that reached epigenome-wide significance were exclusively hypomethylated in the cases, revealing that DNA methylation changes are the most prominent feature of EOPE placentas.

Compared with previous studies, we set a stricter threshold of association of $\mathrm{p}<1 \times 10^{-7}$ to provide a conservative correction. Considering the strict criteria and mild DNA methylation changes (mostly $<10 \%$ ), a large sample size was necessary to ensure statistic power. Meta-analyses are widely conducted to combine genome-wide association study data to improve the power of analysis, but few meta-analyses have been conducted for epigenome-wide association study. In this study, we attempted to minimize the heterogeneity between datasets in the meta-analysis. First, we recruited datasets that sampled placenta specimens from the fetal side and quantified DNA methylation using the Illumina $450 \mathrm{k}$ platform. Additionally, we used cases that were clearly diagnosed with EOPE to reduce phenotypic diversity. Moreover, we used the standardized mean difference of $M$ values as the effect sizes in the meta-analysis, which reduced the batch effects between datasets.

An important confounding factor in EOPE placenta studies is gestational age. The EOPE cases generally involve iatrogenic preterm birth because of severe maternal and/or fetal conditions, resulting in remarkable differences in gestational age between EOPE cases and uncomplicated controls. In some studies, spontaneous preterm controls were employed for gestational age matching. However, some pathological processes overlap between PE and preterm birth, which may reduce the power of detecting relevant DMPs. In contrast, a recent study found a significantly larger gene expression difference between similarly aged preterm controls and PE samples than between term controls and PE samples [4]. Some DMPs identified may have been associated with preterm birth rather than EOPE while using preterm controls. In this study, the datasets recruited in meta-analysis included both uncomplicated and preterm controls, and the DMPs should show a more than 5\% difference between EOPE cases and uncomplicated or preterm controls to ensure that the DMPs are associated with EOPE, rather than with preterm birth or gestational age.

The sample size is critically important in an association study. However, the available data were limited and cannot provide sufficient power to detect association signals for loci with weak effects [29]. Nevertheless, more than 2000 probes showed EWS in this study. More than $90 \%$ of probes were replicated in an independent cohort. These results suggest that large numbers of DMPs have not yet been identified. Widespread loci of DNA hypomethylation 
(A)

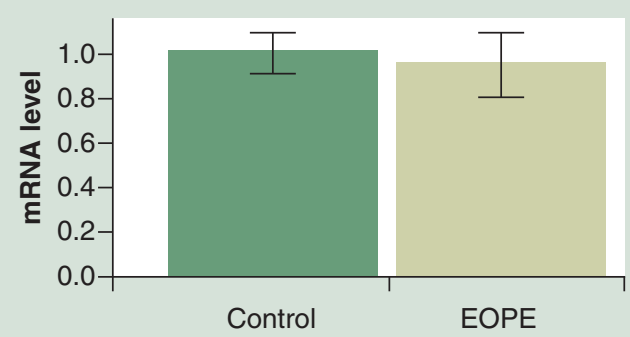

DNMT3B

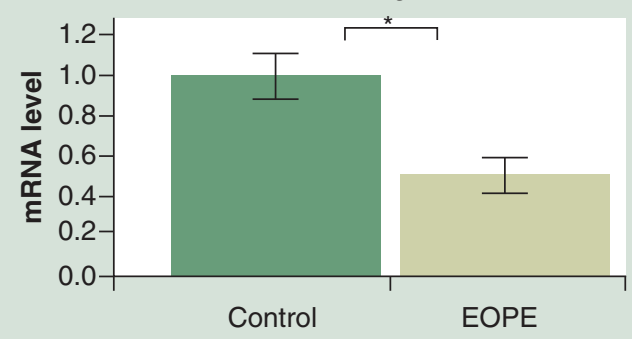

TET2

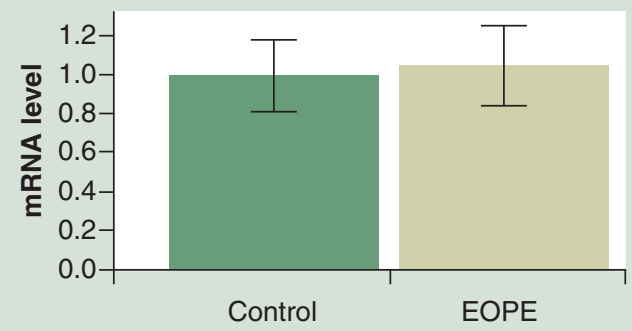

(B)

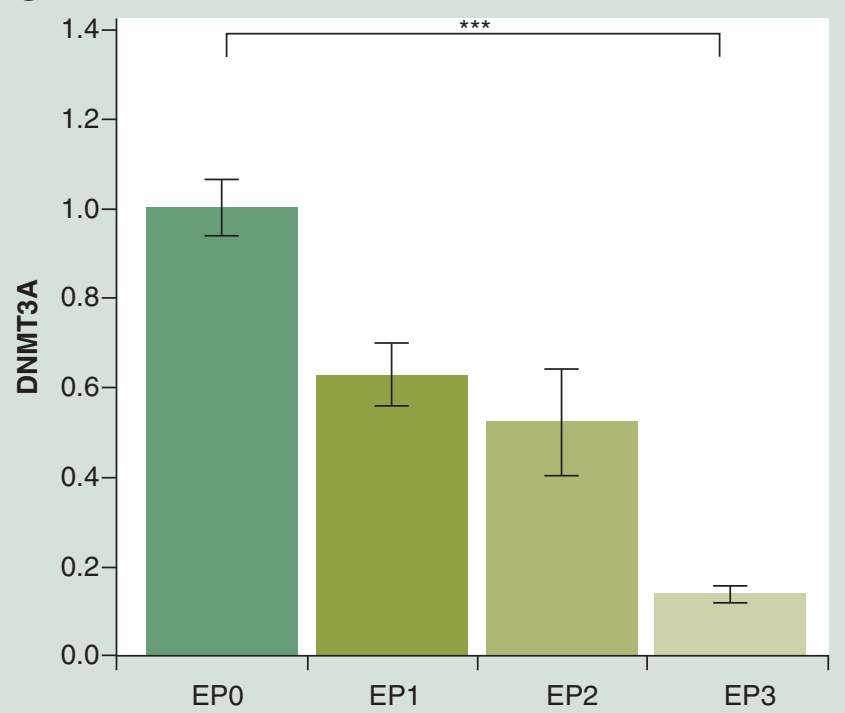

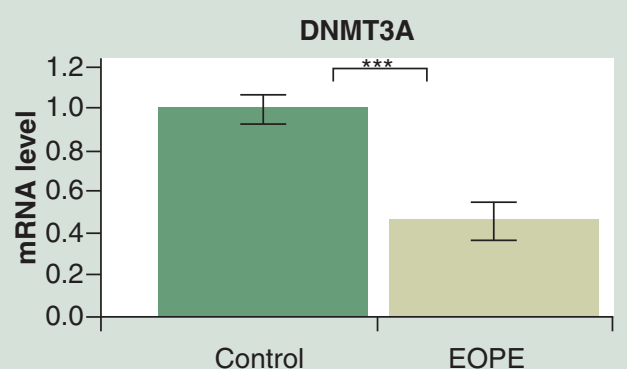

TET1

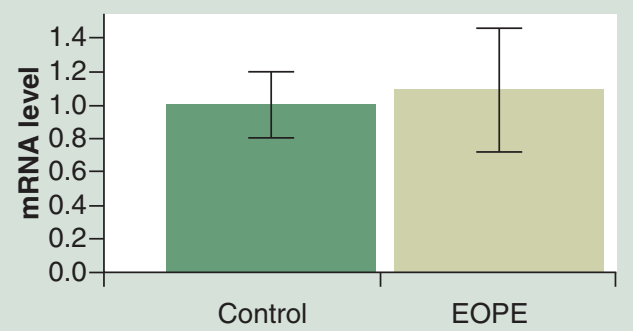

TET3

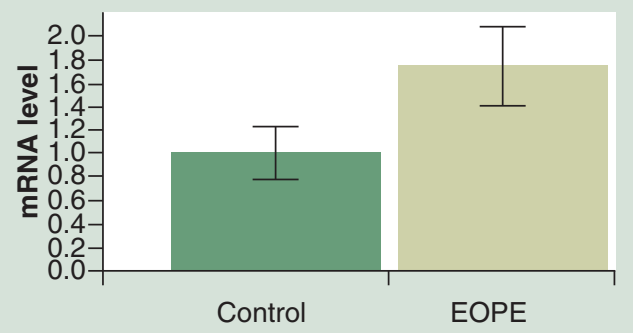

DNMT3B expression of subgroups

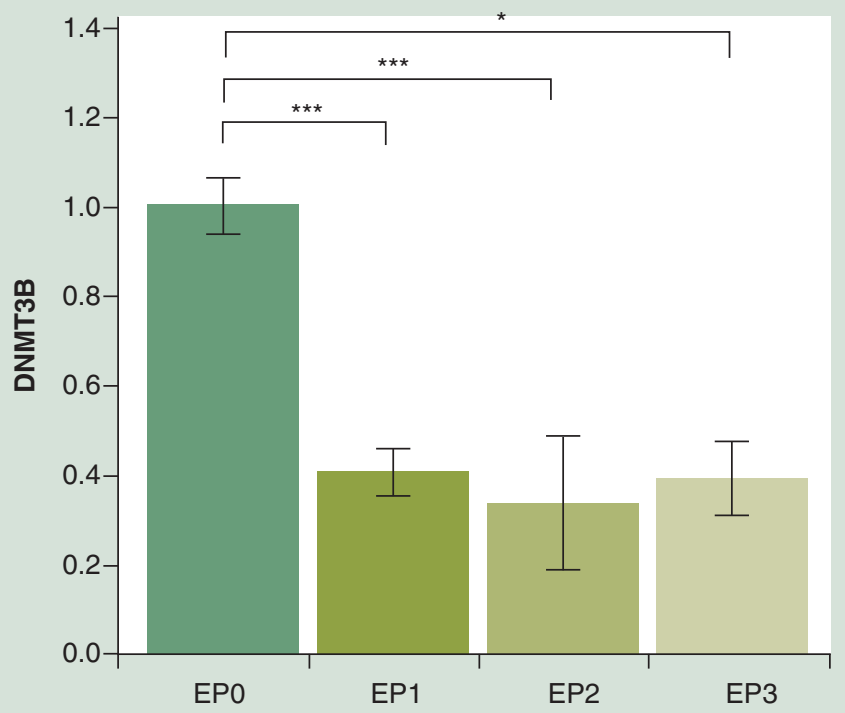

Figure 3. The mRNA levels of three methyltransferases and three TET methylcytosine dioxygenases in placenta. (A) DNMT1 and TET1, TET2 and TET3 expression were not significantly changed in EOPE placenta, and the expression of DNMT3A and DNMT3B were significantly reduced in EOPE placenta control samples are normalized to 1. (B) Compared with samples in EPO, DNMT3A expression in EP3 and DNMT3B expression of samples in EP1, EP2 and EP3 were significantly reduced the expression in EP0 is normalized to 1. ${ }^{*} \mathrm{p}<0.05$; $* * p<0.005 ; * * * p<0.0005$. Error bars: mean \pm standard error. EOPE: Early-onset preeclampsia. 

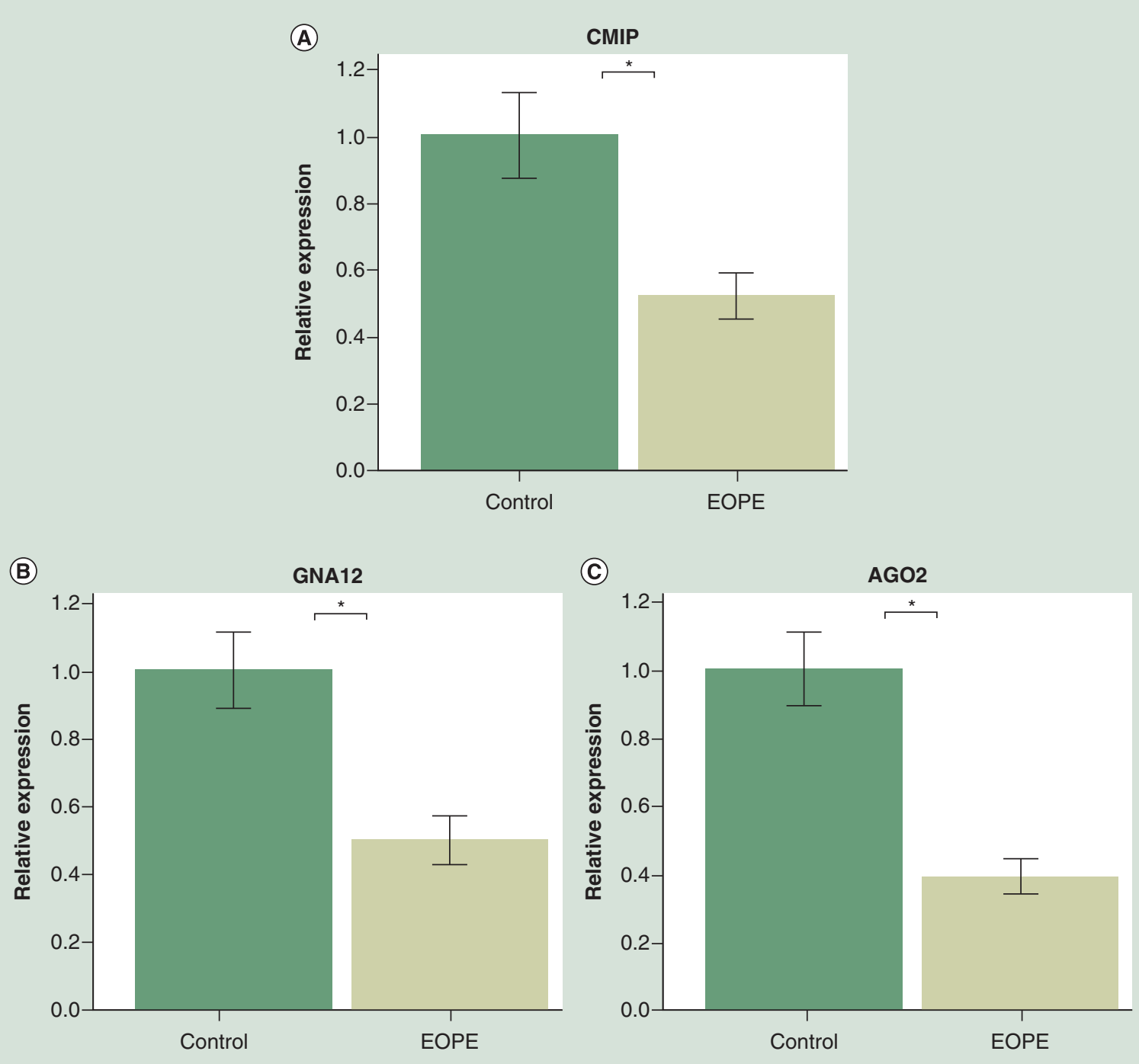

Figure 4. The mRNA levels of four genes in placenta, which covered some of the most significant differentially methylated probes. (A) CMIP, (B) GNA12 and (C) AGO2 were all expressed in lower level. Control samples are normalized to $1 .{ }^{*} \mathrm{p}<0.05 ;{ }^{*} \mathrm{p}<0.005 ;{ }^{* *} \mathrm{p}<0.0005$. Error bars: mean $\pm \mathrm{SE}$.

in EOPE placentas were unlikely to be independent events. Indeed, the downregulated DNA methyltransferases $D N M T 3 A$ and $D N M T 3 B$ in EOPE placentas indicated that DNA methylation changes result from epigenomic metabolic dysfunction.

Hypothetical integration of DNA methylation changes into the pathophysiologic model of PE

DNA methylation is a complex molecular phenotype that is influenced by both genetic and environmental factors. Do DNA methylation changes reflect the fetal genetic predisposition to PE? The placenta is genetically identical to the fetus [30]. A recent genome-wide association study of PE identified only one susceptibility gene (FLT) in the fetal genome, but the association was more significantly associated with LOPE without FGR [31]. Thus, genetic factors likely do not play a causal role in DNA methylation changes. In contrast, the intrauterine environment is a more compelling candidate as responsible for dysfunction of the DNA methylome. Placental ischemia was suggested as the first stage of PE [2], which impacts cellular metabolism such as glycolysis in the placenta. The substrates used for DNA methylation, as well as other epigenetic modifications, are affected by nutrient availability and metabolic pathway activity [32]. We predicted that the DNA methylation changes reflected the epigenetic programming triggered by placental ischemia and resulted in cell state transition to a 'PE' type that released 'toxic' 
factors into the maternal circulation. Therefore, DNA methylation changes may play a role in linking the two stages of PE.

The placenta is composed of diverse cell types of both extraembryonic and embryonic origin. What is the cellular origin of the observed DNA methylation changes? An inclusive criterion for the datasets in this study was that specimens were sampled from the fetal side of the placentas, which were mainly the chorionic plate and villus. A previous study showed downregulated expression of DNMT3A in the syncytiotrophoblast (ST) layer rather than in the villous stroma cells of placentas from patients with PE [33]. If dysregulated DNMTs directly caused DNA methylation changes, these changes also likely occurred in trophoblast cells.

Trophoblasts are epithelial cells that are unique to the placenta [34]. There are three mature subtypes of trophoblasts in the human placenta; cytotrophoblasts (CTs), extravillous trophoblasts (EVTs), and STs. The CTs can differentiate into EVTs and STs. However, EVTs, which anchor the placenta to the uterine wall and remodel the maternal spiral arterioles, are abundant on the maternal side of the placenta. The STs arise through CT fusion in floating villi, which are close to the fetal side of the placenta. Considering the sampling procedures in the available datasets, we predicted that the methylation changes observed in this study reflected abnormal ST fusion in EOPE. Supporting this hypothesis, we observed many DMPs identified in this study in genes involved in trophoblast fusion. In fact, a study showed that hypoxia, which may be caused persistent placental ischemia, impairs trophoblast fusion in vitro [35]. Further studies are necessary to determine the role of DNA methylation in trophoblast fusion and the responses to hypoxia.

Nevertheless, the DNA methylation changes might reflect intersample variation in cellular composition. For example, an excess of immature CT has been observed in EOPE placentas [36]. However, it has also been reported that most DMPs were hypermethylated in ST compared with CT [37]. It is necessary to investigate the cell origins of DNA methylation changes in PE placentas in future studies by purifying subpopulations of trophoblasts. A recent published study found cell specific changes of transcriptome in STs, invasive CTs or endovascular CTs of PE from biopsy specimens of placentas [38], suggesting this strategy may be applied in DNA methylation studies.

\section{Epigenetic diversity in PE \& other clinically relevant obstetrical syndromes}

We observed that the DMPs associated with EOPE clustered the placentas into several clinically relevant groups. The most hypomethylated EP3 group consisted of canonical severe PE cases, which were generally very early-onset with coexisting FGR. In contrast, most samples in the hypermethylated EP0 group were term controls. However, epigenetic programming of the placentas appeared neither specific to PE nor sufficient to cause it, as some isolated FGR cases showed similar epigenetic signatures with EOPE and were clustered into EP2, while a few EOPE placentas were EP0. The LOPE placentas, which were distributed in all groups, showed epigenetic variations. The LOPE accounted for the largest number of total PE cases [39]. It is necessary to understand epigenetic diversity in PE placentas, particularly in LOPE.

The epigenetic diversity of PE may reflect the etiological heterogeneity of this disorder. The PE is diagnosed based on clinical signs in the mother, including hypertension and proteinuria, which are triggered by placental release of soluble factors associated with uteroplacental ischemia. The degree of ischemia is determined by the mismatch between the blood supply of the placenta and fetal growth demand [2]. The fetal and maternal adaptive responses contributed to the clinical phenotypes. Based on the hypothesis that DNA methylation changes reflect epigenetic programming triggered by ischemia, we concluded that severely ischemic placentas (EP3) would develop canonical EOPE with FGR, regardless of the adaptive responses. However, if the ischemia was milder, the fetal and maternal responses would play more important roles in the final phenotypes. Stress induced by ischemia may burst into vulnerable parts of the mothers and/or fetus and result in different phenotypes. Importantly, fetal and maternal adaptive responses may be antagonistic: maternal hypertension may improve uterine perfusion, while fetal growth retardation may reduce the demand for the placental blood supply. The DNA methylation is considered as a reversible and dynamic modification reflecting environmental exposure and therefore may have been responsible for these antagonistic responses in the methylome datasets in this study. For example, mothers who are tolerant of placental releasing factors may not exhibit hypertension to increase uterine perfusion, then resulting in isolated FGR or LOPE with similar placentas to EOPE (EP2). Fetuses insensitive to ischemia may not undergo epigenetic programming, resulting in FGR with placentas similar to those in healthy controls (EP0). In contrast, the mothers or fetuses who were sensitive to ischemia may develop PE superimposed on chronic hypertension or EOPE, increase uterine perfusion, improve fetal growth and rescue epigenetic programming (EP0 or EP1). Moreover, Leavey et al. identified a subset of PE cases with less severe maternal symptoms and evidence of maternal antifetal rejection, 
and the DNA methylome was profiled in the CA2 dataset [24]. The less significant epigenetic programming of this subtype compared with the canonical type suggested that a positive maternal response can resolve methylation changes by restricting fetal growth.

Notably, although some maternal and fetal responses to uteroplacental ischemia appeared contradictory, they may all have fitness (survival and reproductive performance) value under the conditions of proper mother/fetus pairs and be deleterious in maladaptive pairs, which may be explained by the antagonistic pleiotropy theory [40]. For example, a fetus sensitive to ischemia survive better in an insensitive mother, but if the mother is also sensitive, uteroplacental ischemia likely results in severe PE and even cause maternal/fetal death. The coexistence of individuals with different predispositions to ischemia may attenuate or counteract the effect of any related factors in population studies. This may explain the limited success in identifying risk genes or biomarkers for PE [41].

Besides DNA methylation, other epigenetic modifications, in particular microRNA, are involved in the pathophysiology of placental ischemic diseases, including PE [42,43]. Both DNA methylation and miRNA are robust epigenetic marks, which will survive most sample storage conditions [44]. Moreover, cell free nucleic acids released from trophoblast can be stably detected in maternal plasma, and therefore may be noninvasive analyzed in maternal plasma [45].

\section{Conclusion}

The present study indicated that the numerous mild to moderate hypomethylated loci in the placental DNA methylome were associated with EOPE, which may be caused by the dysfunction of de novo DNA methyltransferases and result in abnormal trophoblast turnover. The epigenetic diversity of PE and other pregnancy complications suggests that dynamic and antagonistic responses between mothers and fetuses contribute to clinic outcomes.

\section{Future perspective}

Further studies are required to determine the cell origin of the epigenetic programming in PE by analyzing cell specific DNA methylation. In addition, future research in cell and animal models should also determine if placental ischemia and hypoxia directly lead to DNA methylation changes, and the methylation changes program the placental cells to releasing soluble factors into the maternal circulation. If the intermediate role in linking the two stages of PE would be proved, the identified DMPs may be useful as biomarkers for early diagnosis of PE, quantifying the degree of ischemia, evaluating maternal/fetal responses and classifying the samples for identifying risk genes. Both mothers and children are at an increased risk of chronic diseases after PE exposure [46,47] according to epidemiological studies, but the effect size and penetrance were generally small. The maternal/fetal responses during the onset of PE may also contribute to health and diseases later in life, which would be helpful for applying precise interventions to decrease disease risk.

\section{Acknowledgments}

The authors acknowledge the detail clinical and subgroup information of the CA2 dataset provided by Dr Brian Cox.

\section{Financial \& competing interests disclosure}

This work was supported by the National key research and development program under Grant (grant number 2016YFC1000503), National Natural Science Foundation of China under Grant (grant numbers 81871180 and 81501276 ), the Shanghai Municipal Commission of Science and Technology Program under Grant (grant number 16411968100) and the Shanghai Municipal Commission of Health and Family Planning Program under Grant (grant number 20154Y0039). The authors have no other relevant affiliations or financial involvement with any organization or entity with a financial interest in or financial conflict with the subject matter or materials discussed in the manuscript apart from those disclosed.

No writing assistance was utilized in the production of this manuscript.

\section{Supplementary data}

To view the supplementary data that accompany this paper please visit the journal website at: www.futuremedicine.com/doi/full/ 10.2217/epi-2019-0065 
Summary points

- Studies on differential methylated loci associated with preeclampsia (PE) resulted in poor replicated findings.

- We performed an epigenome-wide association study in a Chinese cohort and published datasets (335 samples in total).

- We identified numerous mild but consistently hypomethylated probes associated with early-onset PE, with 2125 reaching epigenome-wide significance.

- The widespread hypomethylated loci in placenta represented the epigenetic signature of PE.

- The strongest association was observed for $\operatorname{cg} 10246581\left(p=7.2 \times 10^{-26}, \Delta \beta=-0.17\right)$ within CMIP, and three of the top ten significant probes were in this gene.

- The validated differential methylated probes were enriched in for $\mathrm{CpG}$ sites partially methylated and genes related to trophoblast fusion.

- The placentas of multiple pregnancy complications and controls could be clustered into four subgroups by the differential methylated probes: the hypermethylated EPO consisted of mostly healthy controls; the most hypomethylated EP3 exhibited early-onset PE with more severe fetal growth restriction; the EP2 included the less severe early-onset PE; and the intermediate EP1 showed heterogeneous isolated clinical features and antagonistic fetal/maternal outcomes.

- Changes in DNA methylation in PE were associated with downregulated de novo DNA methyltransferases, suggesting that they were a consequence of intrauterine environmental exposure.

- The DNA methylation patterns in PE and other relevant obstetrical syndromes suggested some common pathophysiologies and complex maternal/fetal adaptive responses contributing to variable clinical outcomes.

\section{References}

1. Mol BWJ, Roberts CT, Thangaratinam S, Magee LA, De Groot CJM, Hofmeyr GJ. Pre-eclampsia. Lancet 387(10022), 999-1011 (2016).

2. Chaiworapongsa T, Chaemsaithong P, Yeo L, Romero R. Pre-eclampsia part 1: current understanding of its pathophysiology. Nat. Publ. Gr. 10(10), 466-480 (2014).

3. Ogg G, Chaiworapongsa T, Romero R et al. Placental lesions associated with maternal underperfusion are more frequent in early-onset than in late-onset preeclampsia. Obstet. Gynecol. Surv. 67(3), 154-155 (2012).

4. Leavey K, Benton SJ, Grynspan D, Kingdom JC, Bainbridge SA, Cox BJ. Unsupervised placental gene expression profiling identifies clinically relevant subclasses of human preeclampsia. Hypertension 68(1), 137-147 (2016).

5. Smith ZD, Meissner A. DNA methylation: roles in mammalian development. Nat. Rev. Genet. 14(3), 204-220 (2013).

6. Rakyan VK, Down TA, Balding DJ, Beck S. Epigenome-wide association studies for common human diseases. Nat. Rev. Genet. 12(8), 529-541 (2011).

7. Gao WL, Li D, Xiao ZX et al. Detection of global DNA methylation and paternally imprinted H19 gene methylation in preeclamptic placentas. Hypertens. Res. 34(5), 655-661 (2011).

8. Kulkarni A, Chavan-Gautam P, Mehendale S, Yadav H, Joshi S. Global DNA methylation patterns in placenta and its association with maternal hypertension in pre-eclampsia. DNA Cell Biol. 30(2), 79-84 (2010).

9. Chelbi ST, Mondon F, Jammes $\mathrm{H}$ et al. Expressional and epigenetic alterations of placental serine protease inhibitors: SERPINA3 is a potential marker of preeclampsia. Hypertension 49(1), 76-83 (2007).

10. Wang Z, Lu S, Liu C et al. Expressional and epigenetic alterations of placental matrix metalloproteinase 9 in preeclampsia. Gynecol. Endocrinol. 26(2), 96-102 (2010).

11. Xiang Y, Cheng Y, Li X et al. Upregulated expression and aberrant DNA methylation of LEP and SH3PXD2A in pre-eclampsia. PLoS ONE 8(3), (2013).

12. Blair JD, Yuen RKC, Lim BK, McFadden DE, von Dadelszen P, Robinson WP. Widespread DNA hypomethylation at gene enhancer regions in placentas associated with early-onset pre-eclampsia. Mol. Hum. Reprod. 19(10), 697-708 (2013).

13. Anton L, Brown AG, Bartolomei MS, Elovitz MA. Differential methylation of genes associated with cell adhesion in preeclamptic placentas. PLoS ONE 9(6), (2014).

14. Yeung KR, Chiu CL, Pidsley R, Makris A, Hennessy A, Lind JM. DNA methylation profiles in preeclampsia and healthy control placentas. Am. J. Physiol. Hear. Circ. Physiol. 310(10), H1295-H1303 (2016).

15. Herzog EM, Eggink AJ, Willemsen SP et al. Early- and late-onset preeclampsia and the tissue-specific epigenome of the placenta and newborn. Placenta 58, 122-132 (2017).

16. Wilson SL, Leavey K, Cox BJ, Robinson WP. Mining DNA methylation alterations towards a classification of placental pathologies. Hum. Mol. Genet. 27(1), 135-146 (2018).

17. Martin E, Ray PD, Smeester L, Grace MR, Boggess K, Fry RC. Epigenetics and preeclampsia: defining functional epimutations in the preeclamptic placenta related to the TGF- $\beta$ pathway. PLoS ONE 10(10), (2015). 
18. Januar V, Desoye G, Novakovic B, Cvitic S, Saffery R. Epigenetic regulation of human placental function and pregnancy outcome: considerations for causal inference. Am. J. Obstet. Gynecol. 213(4), S182-S196 (2015).

19. Aryee MJ, Jaffe AE, Corrada-Bravo $\mathrm{H}$ et al. Minfi: a flexible and comprehensive bioconductor package for the analysis of infinium DNA methylation microarrays. Bioinformatics 30(10), 1363-1369 (2014).

20. Morris TJ, Butcher LM, Feber A et al. ChAMP: 450k chip analysis methylation pipeline. Bioinformatics 30(3), $428-430$ (2014).

21. Lehne B, Drong AW, Loh M et al. A coherent approach for analysis of the Illumina HumanMethylation 450 BeadChip improves data quality and performance in epigenome-wide association studies. Genome Biol. 16(1), (2015).

22. Ching $\mathrm{T}$, Song MA, Tiirikainen $\mathrm{M}$ et al. Genome-wide hypermethylation coupled with promoter hypomethylation in the chorioamniotic membranes of early onset pre-eclampsia. Mol. Hum. Reprod. 20(9), 885-904 (2014).

23. Viechtbauer W. Conducting meta-analyses in R with the metafor package. J. Stat. Softw. (2010).

24. Leavey K, Wilson SL, Bainbridge SA, Robinson WP, Cox BJ. Epigenetic regulation of placental gene expression in transcriptional subtypes of preeclampsia. Clin. Epigenetics 10(1), (2018).

25. Huang DW, Sherman BT, Lempicki RA. Bioinformatics enrichment tools: Paths toward the comprehensive functional analysis of large gene lists. Nucleic Acids Res. 37(1), 1-13 (2009).

26. Alexa A, Rahnenführer J, Lengauer T. Improved scoring of functional groups from gene expression data by decorrelating GO graph structure. Bioinformatics 22(13), 1600-1607 (2006).

27. Gerbaud P, Pidoux G. Review: An overview of molecular events occurring in human trophoblast fusion. Placenta 36(S1), S35-S42 (2015).

28. Jones PA. The DNA methylation paradox. Trends Genet. 15(1), 34-37 (1999).

29. Tsai PC, Bell JT. Power and sample size estimation for epigenome-wide association scans to detect differential DNA methylation. Int. J. Epidemiol. 44(4), 1429-1441 (2015).

30. Gude NM, Roberts CT, Kalionis B, King RG. Growth and function of the normal human placenta. Thromb. Res. 114(5-6 SPEC. ISS.), 397-407 (2004).

31. McGinnis R, Steinthorsdottir V, Williams NO et al. Variants in the fetal genome near FLT1 are associated with risk of preeclampsia. Nat. Genet. 49(8), 1255-1260 (2017).

32. Reid MA, Dai Z, Locasale JW. The impact of cellular metabolism on chromatin dynamics and epigenetics. Nat. Cell Biol. 19(11), 1298-1306 (2017).

33. Jia Y, Li T, Huang X et al. Dysregulated DNA methyltransferase 3A upregulates IGFBP5 to suppress trophoblast cell migration and invasion in preeclampsia. Hypertension 69(2), 356-366 (2017).

34. James JL, Srinivasan S, Alexander M, Chamley LW. Can we fix it? Evaluating the potential of placental stem cells for the treatment of pregnancy disorders. Placenta 35(2), 77-84 (2014).

35. Genbacev O, Joslin R, Damsky CH, Polliotti BM, Fisher SJ. Hypoxia alters early gestation human cytotrophoblast differentiation/invasion in vitro and models the placental defects that occur in preeclampsia. J. Clin. Invest. 97(2), 540-550 (1996).

36. Redline RW, Patterson P. Pre-eclampsia is associated with an excess of proliferative immature intermediate trophoblast. Hum. Pathol. 26(6), 594-600 (1995).

37. Yuen RKC, Chen B, Blair JD, Robinson WP, Michael Nelson D. Hypoxia alters the epigenetic profile in cultured human placental trophoblasts. Epigenetics 8(2), 192-202 (2013).

38. Gormley M, Ona K, Kapidzic M, Garrido-Gomez T, Zdravkovic T, Fisher SJ. Preeclampsia: novel insights from global RNA profiling of trophoblast subpopulations. Am. J. Obstet. Gynecol. 217(2), 200.e1-200.e17 (2017).

39. Lisonkova S, Joseph KS. Incidence of preeclampsia: Risk factors and outcomes associated with early-versus late-onset disease. Am. J. Obstet. Gynecol. 209(6), 544.e1-544.e12 (2013).

40. Connallon T, Clark AG. A general population genetic framework for antagonistic selection that accounts for demography and recurrent mutation. Genetics 190(4), 1477-1489 (2012).

41. Benton SJ, Ly C, Vukovic S, Bainbridge SA. Andrée Gruslin award lecture: metabolomics as an important modality to better understand preeclampsia. Placenta 60, S32-S40 (2017).

42. Chiofalo B, Laganà AS, Vaiarelli A et al. Do miRNAs play a role in fetal growth restriction? A fresh look to a busy corner. Biomed Res. Int. 2017, 1-8 (2017).

43. Laganà AS, Vitale SG, Sapia F et al. miRNA expression for early diagnosis of preeclampsia onset: hope or hype? J. Matern. Neonatal. Med. 31(6), 817-821 (2018).

44. Mikeska T, Craig JM. DNA methylation biomarkers: cancer and beyond. Genes (Basel) 5(3), 821-864 (2014).

45. Laganà AS, Favilli A, Triolo O, Granese R, Gerli S. Early serum markers of pre-eclampsia: are we stepping forward? J. Matern. Neonatal. Med. 29(18), 3019-3023 (2016). 
46. Veerbeek JHW, Hermes W, Breimer AY et al. Cardiovascular disease risk factors after early-onset preeclampsia, late-onset preeclampsia, and pregnancy-induced hypertension. Hypertension 65(3), 600-606 (2015).

47. Burton GJ, Fowden AL, Thornburg KL. Placental origins of chronic disease. Physiol. Rev. 96(4), 1509-1565 (2016). 